\title{
MIRADAS SITUADAS SOBRE TRANSLENGUAJE EN UNA ESCUELA EN LA FRONTERA BRASIL-BOLIVIA
}

\section{SITUATED VIEWS ABOUT TRANSLANGUAGING IN A SCHOOL IN THE BORDER BRAZIL-BOLIVIA.}

Ruberval Franco Maciel ${ }^{1}$

Lorene Fernández Dall Negro Ferrari²

\begin{abstract}
RESUMEN: Este artículo busca presentar resultados de una investigación que tuvo como objetivo investigar aspectos de translenguaje que emergieron en la interacción profesora-alunos en el $1^{\text {er }}$ año de la Enseñanza Fundamental de una escuela en la ciudad de Corumbá-Brasil frontera con Puerto Quijarro, Bolivia. La discusión se pautará en autores de la Lingüística Aplicada, sobretodo, en los trabajos de Canagarajah (2013), García y Li Wei (2014) y Rocha y Maciel (2015a,b). Se trata de una investigación de naturaleza cualitativa y exploratoria con características de la epistemología de la emergencia de Sommerville (2008). Los datos fueron recopilados por medio de grabaciones en video y/o audio de las clases, entrevistas con la profesora y observaciones en el diario de campo de la investigadora. El análisis se organizó a partir del concepto de rizoma propuesto por Deleuze y Guattari y resignificado por Marques (2016) y Maciel (2016).
\end{abstract}

PALABRAS CLAVE: Translenguaje. Negociación de sentidos. Transgresión

\begin{abstract}
This paper aims at presenting results of a research that seek to investigate aspects of translanguaging that emerged in the interaction between teacher and students in the first year of Elementary School in a school in the city of CorumbáBrazil in the border with Puerto Quijarro, Bolivia. Discussion will rely on Applied Linguists such as Canagarajah (2013), García and Li Wei (2014) and Rocha and Maciel (2015a, 2015b). It is a qualitative and exploratory research with characteristics of the epistemology of the emergency based on Sommerville (2017). Data were collected through video and audio recordings, interviews with the teacher and field diary obesrvation. The analysis was organized based on the concept of rhizome proposed by Deleuze and Guattari and re-signified by Marques (2015) and Maciel (2016).
\end{abstract}

KEYWORDS: Translanguaging. Meaning making. Transgression.

\footnotetext{
1 Universidade Estadual de Mato Grosso do Sul (UEMS).E-mail: ruberval.maciel@gmail.com

2 Universidade Estadual de Mato Grosso do Sul (UEMS).E-mail: hispanico@hispanico.com.br.
} 


\section{INTRODUCCIÓN}

El contexto contemporáneo de las prácticas de lenguaje, especialmente en zonas de contacto en fronteras geográficas, ha sido cada vez más influenciado por movimientos de diásporas compuestos por hibridismos lingüísticos y culturales. Tal realidad presenta "prácticas dinámicas y fluidas que trascienden las fronteras entre lenguajes, variedades lingüísticas y otros sistemas semióticos", como señala Li Wei (2018, p. 1). Este aspecto ha sido foco de investigación que ha atraído atención de estudiosos del lenguaje sobre el sesgo del translenguaje. En este aspecto, el lenguaje no considera ser visto como algo acabado, pero que sea visto como algo en constante proceso de construcción. En este razonamiento, las teorías nos invitan a repensar cómo el fenómeno del bilingüismo ha sido concebido en los estudios lingüísticos. Desde el punto de vista translingüe, Vogel y García (2017, p. 9) llaman la atención para el hecho de que "en lugar de poseer dos o más sistemas lingüísticos autónomos, como ha sido tradicionalmente pensado, bilingües, multilingües, en verdad, todos los usuarios del lenguaje seleccionan y emplean características particulares de un repertorio lingüístico unitario para hacer sentido y negociar contextos". Resaltamos aquí que el translenguaje es una de las vertientes que ha buscado teorizar el lenguaje no solamente desde el punto de vista de los contextos escolares, como también de escolares como Pennycook (2017), Maciel, Barbosa y Costa (2018).

De los principales precursores de los estudios sobre el translenguaje, se destacan los estudios de Ofelia García (2014) y Suresh Canagarajah (2013, 2017). La autora discurre sobre cuestiones bilingües/translingües en sus investigaciones sobre latinos en escuelas norteamericanas, desde el concepto de translenguaje (translanguaging). A partir del concepto de prácticas translingües (translingualpractice), Canagarajah (2013) defiende una perspectiva de construcción de sentidos más allá del contexto escolar. En Brasil, esa vertiente viene ganando espacio en revistas especializadas y capítulos de libros, como pueden ser vistos en los trabajos de Rocha y Maciel (2015a), Zolin-Vesz (2016) y Lucena y Cardoso (2018), así en disertaciones y tesis, a partir de las investigaciones de Calero Becerra (2018), Dorta (2017), Ferrari (2017), Rupere (2017), Marques (2018), entre otros. Esos estudios buscan resignificar las perspectivas de García y Canagarajah y han dialogado con otras vertientes como, por ejemplo, con el círculo de Bakhtin, con la sociolingüística de la globalización de Blommaert (2010) y con las discusiones sobre superdiversidad (Vertovec, 2007), además de otras.

Las investigaciones desarrolladas por esos autores destacan la complejidad de los procesos de construcciones de sentidos en los diferentes contextos sociales - sean ellos escolares o no escolares. Los estudios, dentro de esa vertiente, traen como objetos de análisis los procesos de translocalización y relocalización por los cuales las diferentes formas de lenguajes y culturas se han transformado. En ese sentido, la superdiversidad es uno de los términos utilizados en la sociolingüística de la globalización. Resaltamos que teorizar sobre la diversidad/superdiversidad no representa una nueva dirección en los estudios del lenguaje. Sin embargo, destacamos que superdiversidad-diversidad enla sociedad digital globalizada contemporánea está cada vez más fuerte. Eso requiere que ampliemos nuestras miradas para las maneras de hacer investigación. En ese razonamiento, Rocha y Maciel (2015b) y Maciel y Ono (2017) señalan la necesidad de diseños de investigaciones que lleven en consideración las lógicas del movimiento, de 
la no linealidad, de la dinamicidad y de la incumplitud como formas también válidas de construir conocimientos en la academia.

Pautados por tales consideraciones y a partir de una mirada situada para el contexto de una escuela de frontera, presentamos, en este texto, un recorte de una investigación cualitativa y de naturaleza interpretivista, organizada de manera rizomática a partir de los datos que surgieron según los preceptos metodológicos de la epistemología de la emergencia postmoderna. La elección por la visión rizomática - resignificada a partir de estudios filosóficos de Deleuze y Guatarri (2011) - se dio porque tal perspectiva no jerarquiza aspectos menos o más importantes. La forma de organización de los datos también se complementa por los fundamentos de la epistemología de la emergencia postmoderna defendida por Sommerville (2008) y Marques (2016). Según esa metodología, la investigación va teniendo forma a partir de los elementos que emergen y, por lo tanto, no tiene como ser previamente pensado a partir de hipótesis, ya que el contexto es desconocido por los investigadores. En otras palabras, el devenir y el inesperado son los aspectos centrales en ese tipo de abordaje en la investigación cualitativa. Tal estudio buscó analizar las prácticas translingües utilizadas en la negociación de sentidos por parte de una profesora y alumnos que tienen raíces en la cultura boliviana. Los datos fueron recopilados en un curso del $1^{\mathrm{er}}$ año de la Enseñanza Fundamental de una escuela pública localizada en la ciudad de Corumbá-Brasil.

Resaltamos que, de los 17 alumnos matriculados en la sala de aula donde fueron recopilados los datos, 14 son hablantes de la lengua española en una escuela brasileña en que la lengua oficial es el portugués. La referida escuela está ubicada en una zona de contacto Brasil-Bolivia. Se trata de un contexto en que las lenguas portuguesa y española se mezclan, así como la cultura de los dos países transita libremente mostrándose, así, como una zona de contacto.

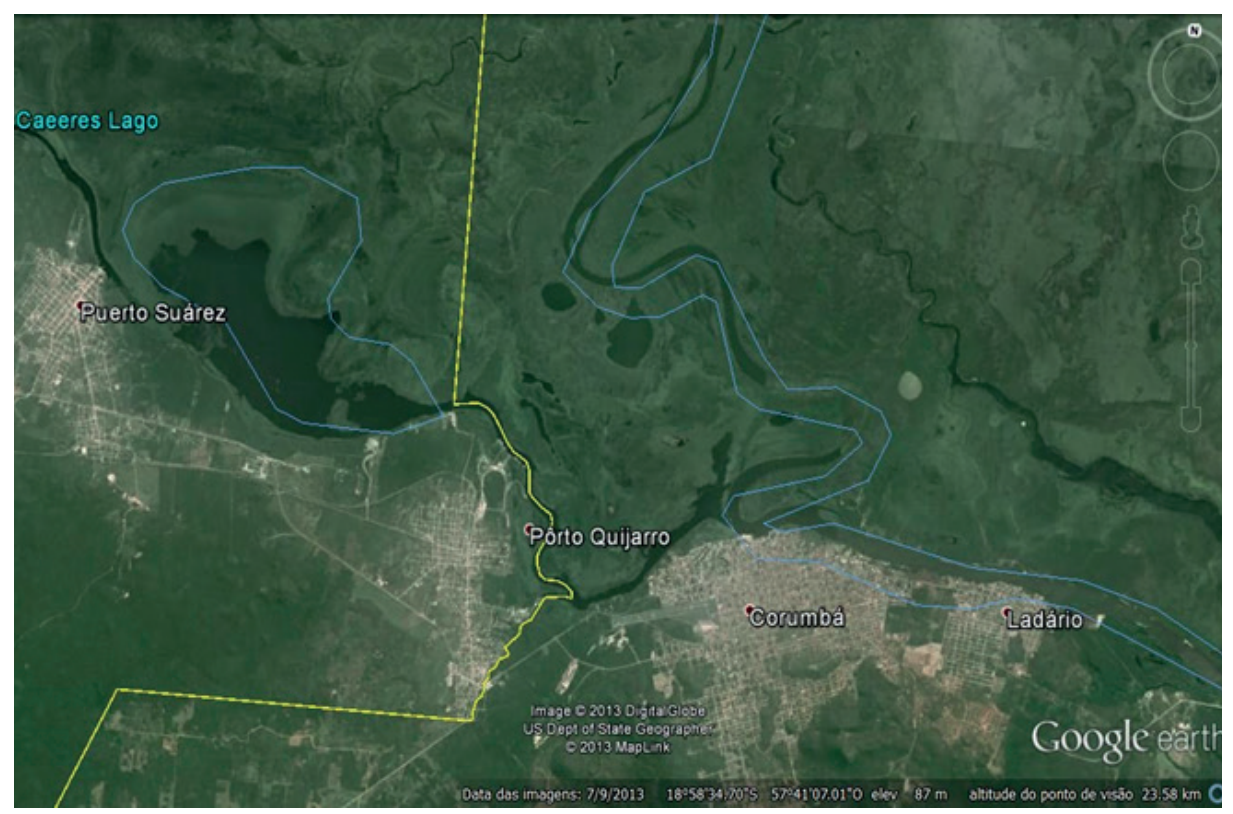

Figura 1 -Vista aérea de la región Corumbá-Puerto Quijarro. Disponible en: http:// www.skyscrapercity.com/showthread.php?t=1661766 
Existe una proximidad muy grande entre las dos ciudades fronterizas, motivo por el cual los habitantes comparten las manifestaciones culturales, el territorio y las costumbres. Solamente un marco territorial divide los dos países. Por lo tanto, es considerada una frontera seca; una frontera dinámica pues brasileños y bolivianos la cruzan a diario por motivo de trabajo, ocio, educación y actividades de las más diversas.

La distancia entre Corumbá con la línea de frontera es de aproximadamente $5 \mathrm{~km}$ siendo un espacio que pertenece al Gobierno Federal de Brasil donde es improbable la ocupación por la población. En contraste, en el lado boliviano, el área urbana de Arroyo Concepción, que compone el municipio de Puerto Quijarro, se acerca a la línea de frontera internacional. El contexto presentado en esta investigación, la frontera Brasil-Bolivia no está acoplada a una concepción estática, un espacio creado por los Estados-nación. Hay una idea de movilidad, de representaciones que componen la identidad y que circulan por ese espacio.

Las peculiaridades existentes en fronteras internacionales son marcadas por diferentes asimetrías de desempeño económico, división geográfica, condiciones sociales y culturales entre los Estados-nación que las componen. En lo que se refiere a la frontera Brasil-Bolivia, escenario de esta investigación, cuando cita Costa (2015), Mancilla (2017) se refiere a grupos sociales y jerarquías creadas y mantenidas por grupos dominantes del lado brasileño donde la desigualdad se manifiesta como juicio de valor despectivo con relación a los bolivianos. Y esa valoración, sigue la autora, se extiende a las áreas económica, da la salud, de la educación, de la seguridad, de la cultura, entre otras.

En ese nuevo territorio la producción de interacción en el sentido de renovar la vida social del grupo en una comunidad desterritorializada, como afirma Mancilla (2017, p. 47), es la recuperación de lo que se entiende como expresión de su esencia, definiendo así la región como una zona de contacto. De esa forma, adentramos en el campo de las manifestaciones culturales y educacionales entre otras.

Consideramos que la teoría del translenguaje como pedagogía puede ser utilizada en diferentes tipos de contextos educacionales y puede desarrollar en los alumnos bilingües/translingües prácticas lingüísticas más flexibles. Al pensar en una pedagogía translingüe, destacamos dos posicionamientos de García y Li Wei (2014) que mencionan la importancia de un trabajo orientado a profesores para el uso del translenguaje en sala de aula. Nos pautamos, todavía, en los argumentos de Adelman, Reyes y Kleyn (2010) y García (2009) que llaman la atención para el hecho de que los profesores en el siglo XXI necesitan estar preparados para ser profesores bilingües en contextos de lenguas en contacto, hecho ese que no ocurre en la mayoría de las instituciones de formación de profesores.

Acerca de ese contexto, Canagarajah (2013) afirma que los alumnos en zonas de contactos lingüísticos enfrentan una batalla ideológica para construir formas efectivas de ciudadanía global y disposiciones cooperativas. En ese mismo razonamiento, Rocha y Maciel (2015) entienden que la diferencia lingüística, identitaria o cultural es vista como más un recurso para la construcción de sentidos, en vez de un obstáculo en el contexto educacional. Ellos defienden que una pedagogía orientada por la idea de prácticas translingües busca preparar los alumnos para aprender a manejar la 
diferencia, con la incertidumbre, con la imprevisibilidad. Apoyados en esas consideraciones, buscamos, por medio de una mirada situada, discutir las prácticas de translenguaje que emergen de la interacción profesora-alumnos en el contexto de enseñanza ubicado en una zona de contacto cuyos alumnos son en su mayoría bilingües.

Zonas de contacto, en el entendimiento de Canagarajah (2013), son espacios donde diversos grupos sociales interactúan y las comunidades son tratadas como menos homogéneas y menos limitadas. Considerando los objetivos de esta investigación de identificar y discutir aspectos relacionados al translenguaje en la interacción profesora-alumno, ilustramos extractos de casos en que emergieron aspectos de translenguaje relevantes. García y Li Wei (2014) abordan la práctica del translenguaje tanto del punto de vista del alumno, translenguaje para aprender, como del punto de vista del profesor, translenguaje para enseñar. Además, García y Li Wei (2014) defienden la importancia del translenguaje para estudiantes de lenguas minoritarias por representar un esfuerzo democrático de promoción de justicia social a través del lenguaje y de la educación.

\section{CUESTIONES EMERGENTES EN EL PROCESO DE NEGOCIACIÓN DE SENTIDOS}

Durante el proceso de recopilación de datos de esta investigación, mudanzas significativas ocurrieron. La producción de un nuevo conocimiento con la emergencia postmoderna, afirma Sommerville (2008), no empieza con la lógica lineal, pero viene de un lugar de no saber, informado por la intuición y capacidad de respuesta. El plan inicial de la investigación, en los términos de Cresswell (2007), no permaneció herméticamente cerrado, y como metafóricamente el autor analiza se forma una trama intrincada como una tela.

Presentamos y discutimos, a continuación, los aspectos de translenguaje que emergieron durante la investigación, los cuales surgen por medio de las prácticas de construcción de sentidos, a través de la recepción adquirida a lo largo de todo el estudio teórico y del análisis del material recopilado. La discusión está organizada en cuatro subsecciones: (1) translenguaje como práctica transgresiva, (2) el uso de las cartillas de alfabetización, (3) metalenguaje por el translenguaje y (4) el translenguaje por la multimodalidad.

\subsection{TRANSLENGUAJE COMO PRÁCTICA TRANSGRESIVA}

Antes de presentar los datos, invitamos al lector a hacer las siguientes reflexiones y consideraciones teóricas: ¿qué competencia traen los translingües para ayudarlos a alcanzar una comunicación exitosa en las zonas de contacto global? Según Canagarajah (2013), en esencia, la competencia translingüe no constituye competencia gramatical, pero sí competencia performativa. De ese modo, el desafío de la adopción del translenguaje es romper conceptos definidos por la orientación monolingüe. Uno de los aspectos que observamos durante las aulas fue la postura de la profesora en transgredir las normas y presupuestos monolingües. En esta subsección, se analiza cada fragmento, buscando la emergencia del translenguaje y los enlaces con las investigaciones de 
Canagarajah (2013) y García y Li Wei (2014).

El motivo por el cual puede ser alcanzado el significado y éxito en la comunicación, en la visión de Canagarajah (2013), es nuestra capacidad de alinear los recursos semióticos con recursos ambientales y sociales. En esa perspectiva, como no existe norma homogénea para significados preconstruidos en zonas de contacto, el autor explica que son requeridas estrategias de negociación adecuadas y prácticas recíprocas.

Según García y Li Wei (2014), los profesores usan el translenguaje para permitir que los alumnos hagan sentido y aprendan. Además, cuando ocurren los momentos creativos y críticos del translenguaje, las acciones son entonces transformadas. El uso de la práctica del translenguaje en las salas de aula, explican los autores, va a explotar la brecha que se forma entre los sistemas monoglósicos de educación de los Estados-nación y las historias locales de los pueblos que tienen una lengua diferente de la definida por el Estado. En la visión de los autores, los contextos y situaciones que surgen en las zonas de contacto provocan una transformación en las nociones de lenguaje tradicionales.

Dado que el término translenguaje fue acuñado originalmente en Galés para enseñar niños bilingües, García y Li Wei (2014) afirman que los estudiosos galeses han sido los primeros que prestaron atención al desarrollo de la pedagogía translingüe. A este respecto, los autores citan Willliams (2012) que distingue la pedagogía translingüe entre natural y oficial. La pedagogía translingüe oficial es conducida y construida por el profesor e incluye más acciones planeadas de los profesores en interacciones con los alumnos. Por otro lado, el translenguaje natural se refiere a actos de los alumnos para aprender, pero puede ser incluido su uso por los profesores con individuos, pares y pequeños grupos. Ante lo expuesto, presentamos aspectos de translenguaje en el siguiente fragmento de interacción ente la profesora y los alumnos.

\section{P: Sí, ¿le quitamos solo? no.Viu \\ P: Tem aqui um. Tijolo. Solo abajo.}

En el extracto arriba, observamos que la profesora conduce el diálogo alternando palabras en portugués y en español para asegurar la plena comprensión de la disciplina en cuestión. En este momento, como propone García y Li Wei (2014), emerge el aspecto translingüe, proporcionando al alumno el desarrollo de nuevos entendimientos y nuevas prácticas lingüísticas.

P. Reta é aquela tirinha que a gente faz com quadradinho. Que é isso: daonde que vc năo sabe o que é a reta numérica?

Al. Él está riendo

P.: Ah! Él ta riendo de mí. ¿Y dónde está tu cuaderno? ¿Lo olvidó en su casa? Voy a procurar en el armario. Que é isso... (Grifos nuestros)

En el extracto ahora transcripto, observamos que nuevamente la lengua española es utilizada por la profesora. Sin embargo, esta vez, ella responde en español al habla de su alumno que utilizó la lengua española también. Diferentemente del aspecto 
analizado anteriormente, ella no estaba buscando hacerse entender. Estaba simplemente haciendo comentarios con su alumno en su lengua. Por lo tanto, esta vez, tanto la profesora como el alumno se valieron de la misma lengua para conseguir comunicación.

Buscamos en las definiciones de García y Li Wey (2014) el entendimiento de las actitudes de la profesora. Los autores recurren a la definición de García y Kano (2010) y explican que en el proceso de translenguaje profesores y estudiantes se empeñan en prácticas discursivas en las cuales son incluidas todas las lenguas de todos los alumnos de la sala de aula. De esa forma, el desarrollo de nuevas prácticas de lenguaje ocurre a la vez que las antiguas son mantenidas.

Los objetivos de la pedagogía translingüe, según García y Li Wei (2014), son acompañados por estrategias translingües que pueden ser realizadas en la educación general o en la educación bilingüe. A partir de esas estrategias, los autores identifican tres categorías: (1) la atención del profesor para hacer sentido por el translenguaje cuando apropiado para la comprensión; (2) el uso por el profesor de recursos de design en la sala de aula para el translenguaje y (3) planeamiento del profesor acerca del currículum y de las estructuras de la sala de aula para el translenguaje. Observamos que la profesora busca, por medio de palabras en portugués, una construcción de sentido, es decir, translenguando cuando apropiado para el entendimiento. En ese sentido, el aspecto translingüe implica en la reconfiguración constante de normas y repertorios, conforme señala Rocha (2015), llevando la profesora a enfrentar innúmeros desafíos constantemente.

\subsection{EL USO DE LAS CARTILLAS DE ALFABETIZACIÓN}

Otro aspecto que nos llamó la atención fue el uso de dos cartillas de alfabetización simultáneas. En una de las clases, en el período destinado a la lectura, la profesora decidió que usaría la cartilla de alfabetización. Puesto eso, buscó en su armario la cartilla llamada Alma de Niño. Confesamos que nos pareció intrigante, pues esa cartilla no estaba escrita en portugués, pero sí en español.



Figura 3. Cartilla de alfabetización en español. 
Conforme la foto y la transcripción a seguir, relatamos el primer diálogo que mantuvimos con la profesora sobre la cartilla.

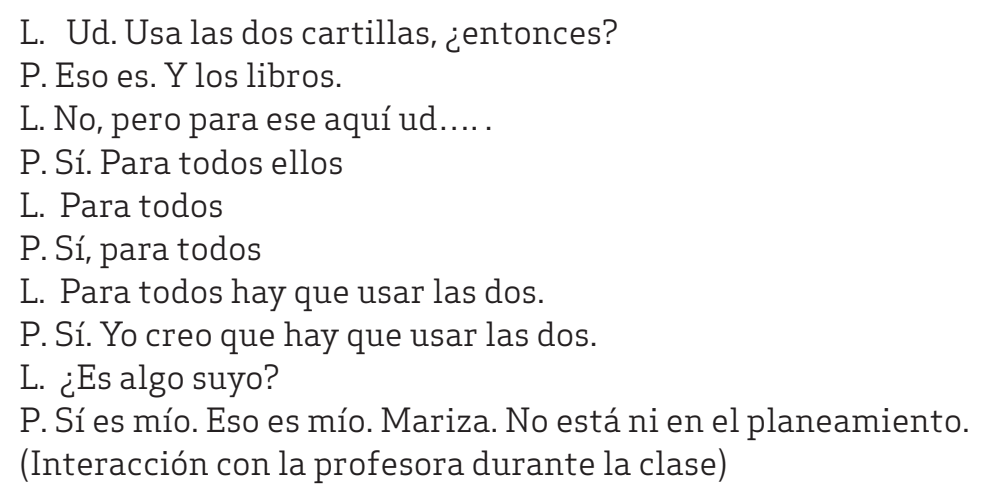

La actitud de la profesora demuestra una práctica que rompe con la norma estándar del proceso educativo vigente. Asociada a la cartilla en portugués, ella utiliza la que está editada en español. Procediendo de esa forma, interpretamos que la profesora posee una postura crítica sobre su hacer pedagógico. Sobre ese aspecto, compromiso es el término utilizado por Canagarajah (2013) para promoción de estrategias y negociación de sentidos. De la misma forma, el compromiso crítico es defendido "en situaciones de uso del lenguaje con base en contextos específicos y objetivos particulares" (ROCHA; MACIEL, 2015a, p.39). Resaltamos que las prácticas translingües representan una postura política y transgresiva. Mientras el resultado de investigaciones sobre las prácticas de translenguaje en contextos escolares no sean más propagadas y validadas por las universidades y por los responsables por las políticas lingüísticas y educacionales, las escuelas públicas del Estado no buscarán crear estrategias nuevas para la realidad que se presenta con relación a los contextos de lenguas en contacto, principalmente en los contextos de fronteras, como es el caso de la presente discusión.

En esa misma dirección, García y Li Wei (2014) afirman que el estudio del translenguaje debería ser implantado en los programas de formación de profesores, caso contrario, profesores y alumnos seguirán utilizando prácticas pedagógicas a partir de la perspectiva monolingüe. En la visión de Rocha y Maciel (2015), cuando pensamos en la orientación translingüe en términos educativos y pedagógicos, ésta redirecciona y resignifica los objetivos y los focos vinculados a la estandarización. De ese modo, a partir de lo que García y Li Wei (2014) y Rocha y Maciel (2015) discuten, asociamos la práctica adoptada por la profesora del uso de una cartilla de alfabetización escrita en español como una forma transgresiva, una vez que la política lingüística de la escuela no preveía esa posibilidad.

Todavía sobre el uso de las dos cartillas, transcribimos el habla de la profesora cuando la investigadora preguntó sobre el uso de la cartilla en espańol.

Con relación al objetivo de yo trabajar la cartilla ahhh Alma de Niño, uno de los principales motivos es porque, ahhh, nosotros no tenemos una gramática que involucre lengua portuguesa y lengua española, específicamente aquí para la frontera. Y Alma de Niño me proporciona lo básico. ¿Tá? Para lectura, para enseñar las primeras letras a los niños. Otro. Otro motivo es porque ahhh tiene textos pequeños que llaman la atención de los niños. Son textos simples, muy simple 
mismos. Un vocabulario propio fronterizo. Y Alma de Niño me lleva a todos los niveles del $1^{\circ}$ al $4^{\circ}$ año. Allá para Bolivia, en el caso del $4^{\circ}$ año. Aquí para la...para en el caso de la frontera. Corumbá. Es muy simple. Pero lo es. Es lo que es más fácil. Es el vocabulario de ellos. Uno de los. (Entrevista realizada con la profesora) (Grifos nuestros)

A pesar de Canagarajah (2013) no referirse al translenguaje específicamente basado en momentos de lectura, una vez que sus análisis se pautan principalmente en la escritura, resaltamos algunas consideraciones. Afirma el autor: "yo construí un ambiente pedagógico favorable, un lugar relativo seguro, para ayudar a los estudiantes a practicar estrategias que todavía traen consigo" (CANAGARAJAH, 2013, p. 135). Observamos que la profesora también buscó ese ambiente favorable. A partir del momento en que el alumno puede leer las primeras letras, las primeras sílabas y las primeras palabras en un contexto familiar, permite a la profesora negociar la construcción de sentido de forma más flexible.

El extracto de la entrevista con la profesora revela la iniciativa de traer lo que era familiar para los alumnos:

\begin{abstract}
El motivo principal es ese. Porque utilizar Alma de Niño. ¿Tá? Tenemos también la facilidad de encontrar esa cartilla. Y muchos padres que tienen hijos aquí en Brasil, ellos trabajan esa cartilla en casa. Yo me di cuenta eso este año, en 2016. Hasta entonces, hasta 2015, 2014 yo no había visto, no había visto. Pero en 2016 me di cuenta que algunos padres, ellos me daban retorno. Y...ellos reforzaban la alfabetización allá en Alma de Niño mientras yo utilizaba los libros adoptados aquí en Brasil. Creo que es por ese motivo.
\end{abstract}

(Entrevista realizada con la profesora)

El feedback de la profesora con relación a la práctica adoptada demuestra surtir efecto cuando relata que muchas veces los alumnos no estudian o no hacen los deberes de casa por causa de la lengua. La mayoría de los padres de esos alumnos no habla portugués, lo que dificulta el auxilio en casa. A partir del momento en que reconocen el material de sus hijos, por estar en la lengua utilizada en casa, ese auxilio se vuelve posible y real muchas veces.

Asociamos la situación presentada con la afirmación de Rocha y Maciel (2015b) en la cual los autores afirman que en el proceso educativo cabe el desarrollo de estrategias para que podamos participar de prácticas de naturaleza tan compleja e inestable. En esa perspectiva, los autores defienden que "el aprendizaje sea un proceso de eterno (re) hacerse, conectado a la performatividad, siendo la atención principalmente hacia el proceso, al cómo, y no al qué" (grifos nuestros). Observamos un alineamiento de los conceptos de Rocha y Maciel (2015a) referidos en el párrafo anterior con la emergencia del translenguaje que surge a partir del proceso utilizado por la profesora. La actitud de la profesora demuestra que vuelve su atención al cómo, en el sentido de promover la construcción de sentidos no preocupándose con el qué.

En el extracto transcripto abajo, identificamos una preocupación de la profesora con relación a la integración de las dos lenguas. En ese sentido, "el translenguaje exige 
un cambio epistemológico en el cual el lenguaje cotidiano de los alumnos y el lenguaje escolar son expandidos e integrados, y al hacerlo, mezcla caminos de saber que son tradicionalmente encontrados en espacios diferentes" (GARCÍA; WEI, 2014, p.146).

P. Vai nesse ou nesse? (la profesora pide para escoger entre la cartilla en portugués y la cartilla en español, ésta es la escogida) Vamos a ver esse. Pa..

Al. Pi - pe-pa papa - pipa pupi.

P. En español que lindo. Sabe dos idiomas. Em português e espanhol. Que rico. Tá? (Grifos nuestros). (En vídeo)

Relatamos, a partir del diálogo transcripto arriba, la alegría por la cual la profesora afirma que el alumno "sabe dos idiomas". De acuerdo con lo que defiende (CANAGARAJAH, 2013, p.188), el translenguaje “se acercará del 'lenguaje estándar' en algunas ocasiones y vernácula en otras, ambas mediadas por los diversos repertorios lingüísticos que los alumnos traen con ellos". De esa forma, entendemos que la sala de aula se vuelve en un ambiente propicio para la convivencia de diferentes culturas, como es el caso de la sala investigada.

Nos respaldamos en Canagarajah (2013) cuando afirmamos que la sala de aula puede ser transformada en un ambiente de socialización, de la misma forma que ocurre en situaciones cotidianas y en contextos sociales. Reflejamos que la práctica translingüe lleva a la socialización entre sus actores, pues, en las palabras de Rocha y Maciel (2015b), busca preparar los alumnos para que aprendan a manejar con la diferencia, con la incertidumbre, con la imprevisibilidad. Tras las reflexiones en relación al recurso propuesto por la profesora, buscamos establecer correlaciones con las estrategias propuestas por García y Li Wei (2014). De ese modo, observamos que en el momento en que la profesora decide utilizar la cartilla de alfabetización en español, emergen recursos translingües refrendados por los autores y que ponderan sobre la producción de un panorama multilingüe multimodal en la sala de aula, incluyendo recursos como textos, palabras en las paredes, recursos auditivos y visuales. Verificamos, entonces, que el recurso de la cartilla se adecua a la propuesta por los autores. Ellos definen aun un panorama textual y visual, así como una propuesta de (bi)letramento. Destacamos aquí que los alumnos están en fase de (bi)letramento y de ese modo aprendiendo a leer en dos cartillas.

\subsubsection{ASPECTOS DE TRANSGRESIÓN}

La cuestión sobre la responsabilidad del Estado por la educación lleva García y Li Wei (2014) a observar que, a partir del momento en que éste asume responsabilidad por la educación de todos los niños, el translenguaje empieza a ser utilizado. Consecuentemente, concluyen los autores, esa práctica será usada para transgredir estructuras educacionales monolingües. A partir de la afirmación de los autores, comparamos la situación con la que encontramos en el lócus de nuestra investigación. En ese caso, la responsabilidad por la educación de todos los nin̄os es de los sistemas educacionales nacionales que, de la misma forma que los autores referencian, imponen 
estructuras monolingües. Sin embargo, la lengua de la mayoría, en el caso investigado, es el español, no la definida por el Estado.

Conforme explican Rocha y Maciel (2015a), el espacio para la contradicción y para el conflicto se abre. De ese modo, los autores se refieren al desafío de que aceptemos impropiedades, entre otras, como nuestras prácticas pedagógicas. En ese contexto, el profesor necesita saber que la diferencia en relación a la orientación monolingüe es que, según Rocha y Maciel (2015a), lo que debe ser apropiado no puede ser impuesto, tiene que ser validado por la práctica. Identificamos ese concepto de los autores en las actitudes de la profesora investigada, cuando decide comprar una cartilla de alfabetización en español. Rompe, así, con las normas vigentes y opta por validar sus conceptos a través de estrategias prácticas. En ese sentido, es necesaria la adopción de prácticas que rompan conceptos de proyectos globales de los Estados nacionales y los de sistemas monoglósicos educacionales.

Según la orientación translingüe, la profesora "transgrede y desestabiliza las jerarquías lingüísticas, y al mismo tiempo amplía y extiende prácticas que son típicamente valoradas en la escuela y en el mundo cotidiano de comunidades y hogares" (GARCIA Y LI WEI, 2014, p. 146). En un extracto de la entrevista que la profesora concedió, nos dijo: "pero es lo que he dicho. Uno molesta. Eso molesta". Se refería ella a sus actos "transgresores" translingües. Canagarajah (2013) defiende que una lente translingüe nos permite romper límites tradicionales. Corrobora entonces con la actitud de la profesora.

Aún sobre la actitud de la profesora por el uso del translenguaje en la escuela, García y Li Wei (2014) defienden su uso en las escuelas cuando se basan en Rosa (2010). Es importante que la escuela abra un espacio de resistencia y justicia social con el empleo del translenguaje una vez que en la escuela puede ocurrir una estigmatización de las prácticas lingüísticas de los jóvenes de minoría. Tras las reflexiones de esta subsección, observamos que la profesora utiliza una práctica diferenciada que busca un abordaje socio crítico y translingüe para que pueda enseñar a sus alumnos bilingües.

\subsection{EL METALENGUAJE POR EL TRANSLENGUAJE}

El flujo multilateral de personas y lenguas que la globalización estimula,conforme explica Canagarajah (2013), ha creado espacios que integran grupos sociales diversos donde las comunidades son tratadas como menos homogéneas y limitadas. A esos espacios, el autor llama de zonas de contacto. García y Li Wei (2014) explican que son zonas donde una lengua es menos entendida como un sistema monolítico autónomo y que ocurre una interacción entre comunidades también.

A partir de las complejas cuestiones lingüísticas que ocurren en las zonas de contacto, (CANAGARAJAH, 2013. p. 57) postula que "la migración involucró personas llevando sus lenguas de herencia para los nuevos locales y desarrollando repertorios que no eran tradicionalmente parte de su comunidad". A partir del concepto de zonas de contactos, García y Li Wei (2014) postulan el translenguaje como 
una forma de capturar las complejas prácticas de hablantes que no pudieron evitar haber tenido las lenguas inscriptas en su cuerpo, y al mismo tiempo vivir entre diferentes contextos sociales y semióticos e interactuar con una compleja orden de hablantes. (GARCIA; LI WEI, 2014, p. 151)

Al considerar los conceptos de los autores, observamos que mismo que las personas se desplacen de sus espacios primeros, que habiten zonas de contacto, sus lenguas están enraizadas en sus cuerpos, no pudiendo así ser arrancadas e inutilizadas. A ese respecto, Canagarajah (2011) explica que el translenguaje puede ser un fenómeno espontáneo cuando se refiere a estudios en contextos escolares. En esas condiciones, relata el autor, en la mayoría de esos casos, actos de translenguaje no son provocados por profesores a través de estrategias pedagógicas conscientes, lo que conforma la espontaneidad del estudio. En la secuencia discutiremos tres aspectos relacionados al translenguaje: (1) aspectos de translenguaje por la pronuncia, (2) negociación de sentidos por el léxico y (3) el translenguaje por la multimodalidad.

\subsubsection{ASPECTOS DE TRANSLENGUAJE POR LA PRONUNCIA}

Uno de los aspectos que no identificamos en las discusiones de autores como García y Li Wei (2014), Canagarajah (2013), Rocha y Maciel (2015a), entre otros, que investigan el translenguaje, fueron aportes relacionados a la pronuncia. Los cambios epistemológicos en la lengua de los alumnos y de la escuela, revelan García y Li Wei (2014), requieren expansión e integración que, cuando concretadas, mezclan formas de saber que son diferentes de aquellas encontradas en espacios tradicionales. En esas condiciones, los autores defienden el desarrollo de prácticas de translenguaje flexibles a alumnos bilingües desarrollando, así, nuevos entendimientos e incluyendo las prácticas estándares. En este estudio, discurrimos a respecto de una práctica que la profesora desarrolló con relación a la pronuncia de los alumnos.

A lo largo de la investigación, notamos que la profesora planea la división de contenidos programados y los divide en momentos. En otras palabras, ella tiene el momento de matemáticas, de escritura, de lectura, así como otros. El momento que destacamos y transcribimos abajo es el conducido por la profesora como momento de lectura de la cartilla de alfabetización en portugués:

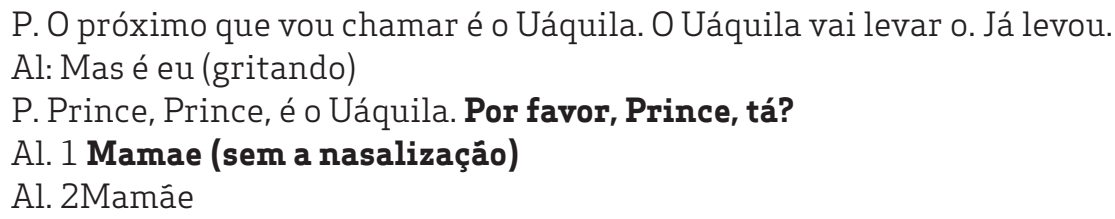

Al leer las palabras destacadas en el extracto arriba, observamos la dificultad del alumno en pronunciarlas. Explicamos aquí que ese alumno tiene como lengua materna el español. La dificultad está en la pronuncia del fonema nasalizado que la palabra mamāe presenta. Eso ocurre porque la nasalización por parte de personas que 
tienen la lengua espańola como materna se torna muy difícil, una vez que no existe en ese vernáculo. García y Li Wei (2014) explican que "además de la maximización de las interacciones de los alumnos y repertorio de significado, el translenguaje incluye prácticas que se encuadran en lo que algunos consideran "lenguaje académico". De ese modo, la corrección de la pronuncia no contrapone los aspectos translingües en esta investigación.

En ese sentido, el desarrollo de una postura crítica frente al modo como las normas se constituyen, en el sentido de favorecer capacidades, estrategias y condiciones de negociación, explican Rocha y Maciel (2015a), es factor relevante para que podamos empeńarnos de manera creativa en situaciones como la descripta en el extracto. Observamos que en este caso la profesora busca habilidades metalingüísticas para identificar, no el significado de la palabra, pero recursos gramaticales para llegar a un acuerdo cuanto a la pronuncia. Habla en "acento circunflexo" e "til"3, hasta mismo habla en un "aaaaa grande".

Interpretamos que la profesora no estaba preparada para esta dificultad y de alguna manera encontró una estrategia dentro de sus posibilidades lingüísticas en ambos los idiomas. En esa perspectiva, retomamos García y Li Wey (2014) cuando explican la realidad del translenguaje natural, o sea, aquella que no es planeada. Como nos muestran Rocha y Maciel (2015a), la diferencia del uso del translenguaje con relación a la orientación monolingüe es que no debe ser impuesto el entendimiento, debe ser validado por la práctica. De esa forma, los objetivos específicos y particulares requeridos son alcanzados, mismo que parcialmente, pero suficientes para el evento investigado.

Identificamos también, en la transcripción anterior, la actuación de un segundo alumno cuando repite la palabra mamâe para auxiliar al compañero. Explicamos que el alumno 2 tiene como lengua materna el portugués. Para explicar esa emergencia, recurrimos a Brantmeier (2005) en lo que se refiere a la construcción de sentido y de participación de los actores que de ella hacen parte cuando menciona el co-learning. De acuerdo con el autor, co-learning construye una "comunidad práctica" más auténtica, provocando así una ruptura en las relaciones de poder en la sala de aula. En ese caso, el alumno 2 estaba desmantelando las relaciones de poder en el momento en que asumió el papel de la profesora. También observamos que la actuación fue aceptada tanto por la profesora como por el alumno que estaba en dificultad, construyendo una comunidad práctica y auténtica.

En lo que se refiere a las prácticas translingües, Canagarajah (2013, p. 190) afirma que "no llevan necesariamente a romper con las reglas gramaticales todo el tiempo". Lo que es importante para el autor es desarrollar una postura crítica con relación a la constitución de las normas y el uso de la lengua en relación con su contexto.

\subsubsection{LA NEGOCIACIÓN DE SENTIDOS POR EL LÉXICO}

Al pensar en el desarrollo de nuevas prácticas de lenguaje para alumnos bilingües, García y Li Wei (2014) señalan que el conocimiento solamente puede ser accedido a

3 Se refiere a acentuación gráfica existente en la lengua portuguesa. 
través de prácticas con que los mismos estén familiarizados. Siendo así, los datos que presentamos en este estudio de caso demuestran que el translenguaje debe auxiliar en la apropiación de un concepto o vocabulario por parte del alumno. De esa forma, según García y Li Wei (2014), el translenguaje permite que los alumnos bilingües entiendan un texto codificado por medio de prácticas a las cuales no están familiarizados.

En un otro momento de la clase, el de matemáticas, la profesora tiene el objetivo de enseñar la operación de resta matemática. Comienza escribiendo en la pizarra las cuentas y explica el método de cómo realizarlas. Atenta a la percepción de sus alumnos cuanto al contenido, descubre que uno de ellos no está entendiendo qué tiene que hacer, lo que demostramos a través de la transcripción del diálogo abajo:

P. As outras você copia tá? Vinte, trinta e dois. Vamos subtrair, ta? Vamo tirar, vamos esconder. Ta bom? Entăo as outras...

Al. Professora

P. Fala amigo. É ta bonito mas ta atrasado ne?

Al (olha firme para a professora)

P. Sí, le quitamos solo? No.Viu. (Grifo nuestro)

En el extracto arriba, observamos que la profesora busca, en su repertorio lingüístico en español, una palabra que signifique la operación pretendida, pues los intentos en portugués no obtuvieron éxito. Observamos que ella hace gestos también, práctica que, asociada al vocabulario, consigue construir sentido y el alumno vuelve a su pupitre para hacer la tarea. Para situar al lector, destacamos que la palabra quitamos, que la profesora utilizó, no sería ideal y sí mermar. Trazamos un paralelo con un ejemplo de García y Li Wei (2014) sobre el uso del translenguaje en una sala de aula de matemáticas. Puntuamos que ni todas las observaciones tienen semejanza con lo encontrado en esta investigación, sin embargo, son relevantes para los objetivos de ésta.

La profesora, en el estudio de García y Li Wei (2014), es bilingüe y el currículo de las aulas de matemáticas fue adaptado para estudiantes bilingües. Por otro lado, la profesora investigada no es bilingüe y el currículo de la escuela no fue adaptado para los estudiantes matriculados. Sin embargo, comparamos el momento en que la profesora norteamericana tradujo una palabra importante que definía el concepto pretendido. En el mismo sentido, la profesora investigada consiguió construir una práctica para obtener el mismo éxito que su colega de Estados Unidos. Hechas las consideraciones, observamos que lo importante para los estudiantes no es solo entender matemáticas, pero también explorar su total repertorio lingüístico por medio de una pedagogía translingüe. De esa forma, la construcción de sentido se hace desarrollando las prácticas lingüísticas que los alumnos traen de casa en paralelo con las prácticas de la sala de aula. De ese modo, aunque considerando algunas diferencias entre las profesoras, la práctica translingüe permitió el éxito de esa acción.

\subsubsection{EL TRANSLENGUAJE POR LA MULTIMODALIDAD}

El concepto de translenguaje implica múltiples estrategias para la construcción de sentido. La noción de esa práctica, según García y Li Wei (2014, p.76), “pone en primer 
plano las diferentes maneras cómo los oradores multilingües emplean, crean e interpretan diferentes tipos de señales lingüísticos para comunicarse". Así, Canagarajah (2013) destaca que la comunicación trasciende las lenguas individuales, también las palabras e implica diversos recursos lingüísticos y semióticos.

Asociamos el concepto de translenguaje propuesto por los autores a la multimodalidad definida por Kress (2010). El autor propone la combinación entre los medios, modos y recursos semióticos para la construcción de sentido. La comunicación entre dos actores conforme afirma Canagarajah (2013, p. 11), refleja "nuevos modos comunicativos a la medida que las personas adoptan estrategias creativas para relacionarse y representar sus voces". En esta subsección, presentamos un diálogo de la profesora con el alumno que demostraba dificultad para entender la tarea propuesta. Matriculado desde el inicio del año, vive con su familia en Bolivia y la lengua en que se comunican es el espańol. No es una situación impar en la sala de aula investigada, pues una gran parte de los alumnos tiene esa característica. Sin embargo, lo que hace peculiar es que ese alumno no hablaba nada. Hace pocos meses empezó a hablar, pero solamente en espańol. Transcribimos aquí el habla de la profesora:

Él aprendió a hablar. Él no hablaba nada. Solamente se quedaba así (apunta al alumno). Lloraba. Porque él no sabía hablar con nosotros. Él descubrió que habla. Que él habla hace poco tiempo. Unos 4 meses, 5 meses." (Interacción con la profesora, durante la clase).

A partir del relato de la profesora, interpretamos que demuestra aspectos que van más allá de la modalidad oral. La preocupación de ella con ese alumno viene al encuentro con una de las metafunciones que Flores y Woodley (2012) documentaron y que García y Wei (2014) relatan: la creación de vínculos afectivos con los alumnos. La profesora comenta que, con mucho cariño y paciencia, consiguió con que el pequeño comenzara a decir palabras y de esa manera descubrir que tenía el don del habla también. Se preocupa la profesora, con el motivo de esa dificultad, pero, de cualquier forma, él ahora habla. Transcribimos, a continuación, fragmentos de la conversa de la profesora cuando se refiere al habla del alumno:

L. ¿Ese que no hablaba?

P. No hablaba. Y ahora, es el que... cuenta la historia. Él la inventa. Él hace. Él no tiene más miedo.

L. Él habla bien el español.

P: No. Él solamente habla el español.

P: Cuando descubrió que él conseguía hablar él se sintió así, hablador. Y así fue. (Interacción con la profesora durante la clase) (En video)

Delante de las dificultades presentadas con relación a la comunicación entre la profesora y el alumno, recurrimos a García y Li Wei (2014, p.76) al afirmar que "las interacciones multilingües exitosas siempre fueron auxiliadas por las multimodalidades: gestos, objetos, señales visuales, toque, tono, sonidos y otros modos". Presentamos la interacción entre profesora y alumno en el diálogo abajo: 
P. Aqui já viu. Tem um jota de tijolo. Né? E aonde mais?

Al. 1. Aponta sem falar

P. Aqui ta escrito mala. Mala tem jota? Dónde está jota?

Al. 2: Cajú.

Al.1: Aponta sem falar

P. Aqui encima tem, mas aqui năo. Aqui tem. Aqui tem 1,2,3,4,5 jotas.

Al.2. Mas é só aqui embaixo.

P.: Aqui é. Só aqui embaixo. Agora mostra pra mim.

Al. 2. Sóaqui.

Al. 3. Solo la abajo.

P. Tem aqui um. Tijolo. Solo abajo. Năo, năo su pie. Há! Há! Năo vai dar chute nele năo. Marca pra mim.

Al.1. Aponta.

P. Isso. Caju. Caju.

Al.2. Aqui já foi. Aqui já foi.

P.: Aqui já foi. Aqui já foi. Ainda tem mais um jota. Aonde mais tem jota?

Al.1 Pensa, em dúvida.

\section{P. Dónde está más un jota?}

Al. 1. Calado e aponta.

P. Eh! Eh! Palmas. Olha só. Tijolo tem jota. Jota. Jipe tem jota. Caju tem jota. Tá? Mala náo tem. Peteca. Peteca. Náo é peteca. Peteca.

Al. 3 Peteca.

Al. 2 Petêca.

P. Peteca. Pe- te- ca. É para jogar. Mas é só com jota, tá? Você vai marcar pra mim lá na sua carteira. Va en su silla. Por favor.

Al. 1 Primero las dos jotas.

P. Eso, solo que temun jota. ¿Listo? Sí, de tres. (Grifos nuestros)

En ese momento de la clase, cada alumno tenía una tarea diferente, pues era necesario que terminaran todas las actividades propuestas para completar la carpeta y entregar a los padres al final del semestre. La tarea, que el alumno investigado no había hecho, tenía el objetivo de enseñar la letra "j" y las palabras que contenían esa letra.

A lo largo del diálogo, observamos que la profesora intenta de todas las formas explicar el objetivo de la tarea. La estrategia de utilizar la lengua española en conceptos y vocabulario clave remete a García y Li Wei (2014) cuando se refieren a la estrategia de la atención del profesor para construir sentidos por el translenguaje. En ese sentido, la profesora reconoce que debe buscar otros recursos, creando así otras posibilidades de éxito en la construcción del entendimiento de la tarea.

Asociando el uso de palabras en español, la profesora intenta la comunicación de modo gestual. Mueve las manos y señala las letras y palabras constantes del texto. Para ampliar la posibilidad de dar sentido a sus gestos, recurre a las expresiones faciales y una postura delicada y de paciencia. Destacamos el momento en que la profesora dice "ahora muestra para mí", indicando un acercamiento al alumno, creando un ambiente propicio para contornar la dificultad y la presión que implica la tarea.

Al utilizar nuestras capacidades receptivas, Canagarajah (2013, p.14) afirma que "podemos comprender el lenguaje del interlocutor de la misma forma que el interlocutor utiliza su competencia para comprender nuestra propia lengua". En ese sentido, resaltamos la actitud del alumno que también recurre a la competencia gestual en el 
momento en que demuestra su duda a través de una mirada muy profunda como que enviando el recado de que no había entendido.

Por fin, el alumno demuestra que consiguió entender la tarea cuando señala la palabra correcta y la profesora aplaude, en el sentido de elogio y demostrando el acierto de la tarea. En ese sentido, Canagarajah (2013) nos apunta que los signos lingüísticos múltiples que son considerados por la semiótica multimodal están a la disposición de los creadores de signos, haciendo parte de un repertorio de recursos modales. Concluye el autor que, el translenguaje abarca todos los modos de hacer sentido.

\title{
CONSIDERACIONES FINALES
}

\author{
P: Caderno de matemática. Onde está tu cuaderno? \\ L. Vc usa as duas cartilhas, entăo? \\ P. Sim. Pra todos eles
}

En español que lindo. Sabe dos idiomas. Em português e espanhol. Que rico. Tá? Sí, ¿le quitamos solo? no.Viu

Tem aqui um. Tijolo. Solo abajo (Grifos nuestros)

Iniciamos las consideraciones finales con recortes de las hablas de la profesora investigada que trajeron a este trabajo emergencias relevantes. Observamos en esas palabras situaciones que nos llevaron a descubrir que la enseñanza en zonas de contacto tiene que ser revisada. A partir de los datos que emergieron durante el proceso de recopilación, se crearon categorías siguiendo un orden rizomático que no jerarquiza aspectos más o menos importantes. En lugar de ello, destaca los aspectos que emergieron y que podrían establecer un diálogo con la perspectiva translingüe. Enfatizamos como aspecto positivo la actitud transgresora de la profesora que, por ese motivo, hizo con que emergieran prácticas translingües que desconocía y mostrar que la justicia y práctica social también hacen parte de la enseñanza y de regiones de contacto.

A partir de las categorías que han emergido, observamos algunas semejanzas con los aspectos traídos por García y Li Wei (2014) y Canagarajah (2013) en sus estudios. Destacamos el momento en que la profesora y alumnos incluyen las dos lenguas en sus prácticas discursivas y cuando ella negocia sentidos a través del léxico. Sin embargo, algunos aspectos como el uso de dos cartillas de alfabetización simultáneas y la corrección de pronuncia que están entre las categorías definidas en esta investigación, no fueron encontrados en nuestras lecturas.

Las discusiones provocadas por la enseñanza a través de prácticas pedagógicas translingües, con objetivos de enseñar y aprender, en el entendimiento de Rocha y Maciel (2015a), implican en innúmeros desafíos. Sin embargo, esos desafíos, según García y Wei (2914), demuestran el potencial que la pedagogía translingüe tiene que desarrollar: un discurso más sofisticado, profunda comprensión de texto, reconocimiento de lo que los estudiantes realmente saben y también las voces de los alumnos de las lenguas minoritarias. En lo que se refiere a las zonas de contacto, Rocha y Maciel (2015a) relatan que no hay una única y estable variedad estándar, lo que implica en el desafío de que convivamos con la norma, reacentuando esos discursos autoritarios, en 
el medio a la diversidad, donde los estudiantes serán capaces de construir sentido a través de sus lenguas y sus culturas y concluir sus aprendizajes de forma substancial.

Apoyados en los pensamientos de García y Li Wei (2014), Canagarajah (2013) y Rocha y Maciel (2015a), señalamos que las escuelas de las regiones donde las culturas y las lenguas se mezclan, las llamadas zonas de contacto necesitan de políticas propias pues reciben alumnos con lenguas y culturas diferentes. García y Li Wei (2014) resaltan que es necesario que los profesores se vean construyendo y desarrollando las lenguas adicionales de los alumnos mientras los educan. De la misma forma, Rocha y Maciel (2015a) defienden que el translenguaje puede abrir espacio para perspectivas no delimitadas por un concepto binario y por relaciones menos colonizadoras en términos de lengua y cultura.

A partir de las lecturas y apuntes que hicimos, observamos que el universo que implica la práctica del translenguaje es muy amplio. García y Li Wei (2014) relatan que una enseñanza en mutación, donde las lenguas minoritarias están ganando visibilidad a través de políticas escolares que atienden a esas necesidades. Por lo tanto, reconocer que la lengua no es un sistema estable y coherente y mirar a través de diferentes posiciones, pero no dejando de lado los discursos normalizadores, es un desafío que nos lleva a romper con límites tradicionales y expresar la igualdad en las zonas de contacto, conduciendo a un relacionamiento igualitario entre sus habitantes.

Hechas tales consideraciones, llamamos la atención para el hecho de que las interpretaciones seńaladas a lo largo de este artículo son contingentes, situadas y que son pasibles de otras interpretaciones, dependiendo del lócus de enunciación del lector de este texto.

\section{REFERENCIAS}

ADELMAN REYES, S.; KLEIN, T. Teaching in two languages: A guide for K-12 bilingual educators. Thousand Oaks, CA: Corwin Press. 2010New York: Cambridge University Press. 2010.

BLOMMAERT, J. The sociolinguistics of globalization. New York: Cambridge University Press. 2010.

BRANTMEIER, E.J. Empowerment pedagogy: co-learningandteaching.Indiana University. 2005. Disponível em: http://www.indiana.edu/-leeehman/brantmeier.pdf. Acesso em: mar. 17.

CALERO BECERRA, J.A. Negociaçâo de sentidos de línguas de sinais por meio do uso do abecedário datilológico: um olhar situado sobre as práticas translíngues. 2018. Dissertaçăo (Mestrado em Letras). Mestrado em Letras: Universidade Estadual de Mato Grosso do Sul, Campo Grande, 2018. 
CANAGARAJAH, S. Codemeshing in Academic Writing: Identifying Teachable Strategies of Translanguaging. The Modern Language Journal, 95 (3), p.401-417, 2011.

CANAGARAJAH, S. Translingual Practice: Global Englishes and Cosmopolitan Relations. London: Routledge, 2013.

CANAGARAJAH, S. Translingual Practices and Neoliberal Policies: Attitudes and Strategies of African --Skilled Migrants in Anglophone Workplaces., Switzerland: Springer International Publishing, 2017.

CRESWELL, J.W. Qualitative inquiry and research design: Choosing among five approches.Thousand Oaks, CA: Sage, 2007.

DELEUZE, G.; GUATTARI, F. Mil Platôs: capitalismo e esquizofrenia. Traduçăo de Ana Lúcia de Oliveira, Aurélio Guerra Neto e Celia Pinto Costa. Săo Paulo: Editora 34, vol.1, $2^{\text {a }}$ ed. 2011.

DORTA, J. V. Palavreando: uma proposta de aplicativo educacional para aaprendizagem de português escrito pelos surdos Dissertaçâo (Mestrado em Linguística Aplicada). Metrado em Letras: Universidade Estadual de Campinas, Camoinas, 2017.

FERRARI, L.F.D.N. En Español! Qué lindo! Sabe dos idomas! Em português e Espanhol. Que rico, tá? Um olhar situado sobre aspetos de translinguagem na interaçâo professora/alunos em uma escola de fronteira de Brasil-Bolívia. Dissertaçâo ( Mestrado em Letras),Universidade Estadual de Mato Grosso do Sul, Campo Grande, 2017.

GARCIA, O. Bilingual education in the 21th century: A global perspective. Oxford: WileyBlackwell, 2009.

GARCÍA, O., FLORES, N., E WOODLEY, H. Transgressing monolingualism and bilingual dualities: translanguaging pedagogies. In A. Yiakoumetti (ed.), Harnessing linguistic variation to improve education. Bern: Peter Lang, pp. 45-75, 2012.

GARCIA, O.; WEI, L. Translanguaging: Language, Bilingualism and Education. London: Palgrave Macmillan, 2014.

KRESS, G. Multimodality: A social semiotic approach to contemporary communication. London: Routledge. 2010.

LUCENA, M.I.P; CARDOSO, A. Translinguagem como recurso pedagógico: uma discussāo etnográfica sobre práticas de linguagem em uma escola bilíngue. Calidoscópio, v. 16, p. 143-151, 2018. 
MACIEL, R.F. Por outras epistemologias de pesquisa em formaçăo de professores. In: MARQUES, N. Da formaçâo continuada de professores aos momentos de tensâo em sala de aula: Rizoma, Emergência e Letramentos. Campinas: Pontes Editores, 2016.

MACIEL, R.F.; BARBOSA, D.A.; COSTA, M. Translanguage, multimodality and learning in medical spaces: discussing meaning making processes (paper presentation). In: 6th Conference on Multicultural Discourse. Tilburg - The Netherlands: The University of Tilburg. 23-25/10, 2018.

MACIEL, R.F; ONO, F.T.P. Desaprendendo para aprender: questionamentossobre e para a formaçăodocente. (Prefácio). In:SANTOS, L.I.F; MACIEL, R.F.; FORTILLI, S.C. (orgs.) Formaçâo docente e ensino de Língua Portuguesa: resultados e perspectivas do/no Profletras - Regiăo Centro-Oeste. Sinop: Editora Unemat, 2017.

MANCILLA BARREDA, S. V. Interculturalidades no contexto Puerto Quijarro (Bolívia)Corumbá (Brasil). Português língua de fronteiras: ensino, aprendizagem e formaçâo de professores. (Tese Doutorado - Programa de Pós-Graduaçăo em Educaçâo) Universidade de Săo Paulo, Săo Paulo, 2017.

MARQUES, N. Da formaçâo continuada de professores aos momentos de tensâo em sala de aula: Rizoma, Emergência e Letramentos.Campinas: Pontes Editores, 2016

MARQUES, A. N. Práticas translíngues e colaborativas em um curso de inglês. (Tese de doutorado em Letras). Universidade Federal do Paraná, Curitiba, 2018.

PENNYCOOK, A. Translanguaging and semiotic assemblages. International Journal of Multilingualism, 14:3, 269-282, 2017.

ROCHA, C.H. Ensino de língua inglesa na contemporaneidade: diálogos entre prática trasnlingue e a teoria bakhtiniana. In: ROCHA, C.H.; BRAGA, D.B.; CALDAS, R.R. (Orgs.). Políticas linguísticas, ensino de línguas e formaçâo docente: desafios em tempos de globalizaçăo e internacionalizaçăo. Campinas: Pontes, 2015.

ROCHA, C.H.; MACIEL, R.F. Ensino de língua estrangeira como prática translíngue: articulaçōes com teorizaçōes bakhtinianas. D.E.L.T.A., 31-2. 2015 (411-415), 2015a.

ROCHA, C.H.; MACIEL, R.F. Língua Estrangeira, formaçăo cidadă e tecnologia: ensino e pesquisa como participaçăo democrática. ROCHA, C.H.; MACIEL, R.F.. Língua estrangeira e formaçâo cidadâ: por entre discursos e práticas. Campinas: Pontes, $2015 b$.

RUPERE, T.T. Processos de translocalizaçâo e relocalizaçâo da imigraçâo oriental em Campo Grande-MS: discutindo aspectos de translinguagem, metrolinguagem e transculturalidade. (Dissertaçăo de Mestrado em Letras). Universidade Estadual de Mato Grosso do Sul, Campo Grande, 2017. 
SOMMERVILLE, M.J. Waiting in the chaotic place of unknowing: articulating postmodern emergence. In: International Journal of Qualitative Studies in Education. Vol. 21, n. 3, p. 209-222,2008.

VERTOVEC, S. Super-diversity and its implications. Ethnic and racial studies. Vol. 30, n. 6, P. 1024-1054, 2007.

VOGEL, S.; GARCIA, O. Translanguaging. In: NOBLIT, G.; MOLL, L. (Eds.). Oxford Research Encyclopedia of Education. Oxford: Oxford University Press, 2017.

WEI, L. Translanguaging as a Practical Theory of Language. Applied Linguistics, Volume 39, Issue 1, Pages 9-30, 1 February 2018.

ZOLIN-VESZ, F. Por uma pedagogia translíngue para o ensino de línguas. In: JESUS, D. M.; CARBONIERI, D. (Org.). Práticas de Multiletramentos e Letramento Crítico: outros sentidos para a sala de aula de línguas. Campinas: Pontes, 2016. 